\title{
Review of Ten Years' Experience of Endoscopic Skull Base Surgery in A Regional Hospital ENT Department - Queen Elizabeth Hospital
}

\author{
Chong Hou Ming Leo* \\ Department of Ear, Nose, Throat Surgery, Queen Elizabeth Hospital, China
}

*Corresponding author: Chong Hou Ming Leo, FHKAM (Otorhinolaryngology), Chief of Service, Department of Ear Nose Throat

Surgery, Queen Elizabeth Hospital, China

\begin{abstract}
Endoscopic skull base surgery is a relative new approach in ENT specialty. ENT Department of Queen Elizabeth Hospital started to do some ten years ago. It is a review of the types and experience of that endoscopic transnasal approach to manage skull base disease. 63 procedures in four types of surgery is recorded in a ten years period in that regional hospital in Hong Kong. The largest number being done is endoscopic nasopharyngectomy which is more common in southern part of China.
\end{abstract}

\section{Introduction}

The endonasal and endoscopic approach to the skull base provides a minimally invasive way to remove tumors that would otherwise require either a large cranial opening and brain retraction or disfiguring facial scars. Instead, an endoscope is advanced through the natural opening of the nasal cavity. A variety of tumors can be removed in this fashion, in various locations, with different maneuver of the endoscope to the desired location. This approach was first developed by endoscopic sinus surgeons but is now being used as minimally invasive skull base surgery to treat tumors as well. A ten years review of the cases being done in a regional hospital in Hong Kong gives some information about the possible future development of this field of surgery.

\section{Method}

Cases review of hospital notes and operative records from 2010 to 2019 in ENT Department of Queen Elizabeth Hospital. Different surgery involved skull base lesion done with endoscopic endonasal approach were studied.

\section{Result}

Four types of endoscopic endonasal skull base surgery were being done a) Endoscopic nasopharyngectomy about 54 procedures done for recurrent NPC and result like open surgery.

b) Five procedures done for CSF leak \& one is repeat procedure.

c) Two intranasal neuroblastoma \& 1 sphenoid sinus procedures done for inverted papilloma.

d) Two intraorbital and intraconal haemangioma removal procedures.

\section{Discussion}

The development of navigation system and other power instrument used endoscopically helped the development of endoscopic skull base surgery to be done more safely and effectively. Example of endoscopic nasopharyngectomy was done first in ENT department QEH in 2010 for a post radiotherapy nasopharyngeal carcinoma patient with primary adenocarcinoma. It was done with two surgery four hands technique (Figure 1) and a complete excision was achieved. Subsequent endoscopic nasopharyngectomy were done for recurrent NPC patients with aid of power instrument of coblator which give a thin cut layer by layer when approaching 
the dangerous area of carotid and skull base (Figures 2\&3) A nasoseptal flap were created to cover the nasopharyngectomy wound and help patient recovery faster (Figure 4). The overall longterm result is about the same as open surgery. The second types of surgery done for skull base lesion is cerebrospinal fluid leak which either resulted from iatrogenic causes or spontaneous causes or from meningocele. Dura seal and fibrin glue and cauterization of meningocele were used to stop the CSF leak. Lumbar drain was used by neurosurgeon in these cases when two specialty worked together (Figures 6 \& 7).

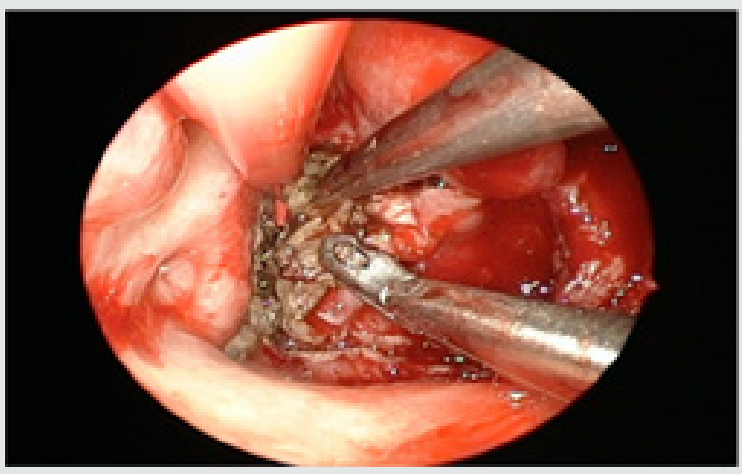

Figure 1: Two surgeon four hands technique.

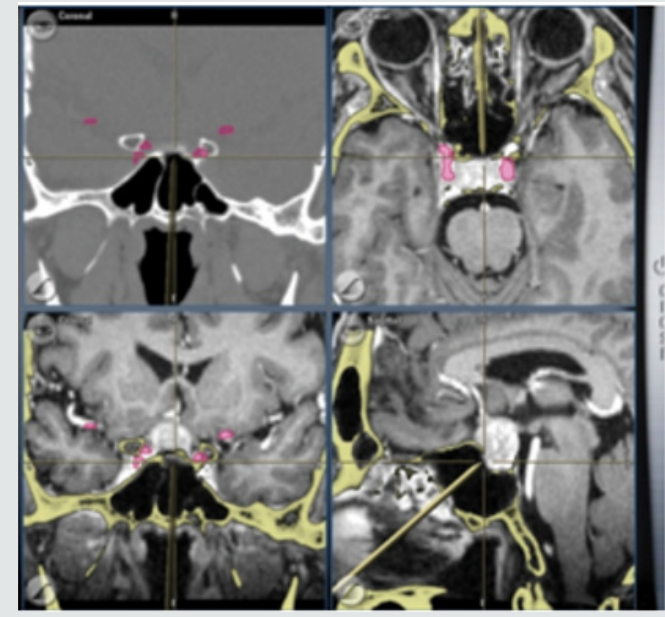

Figure 2: Navigation system.

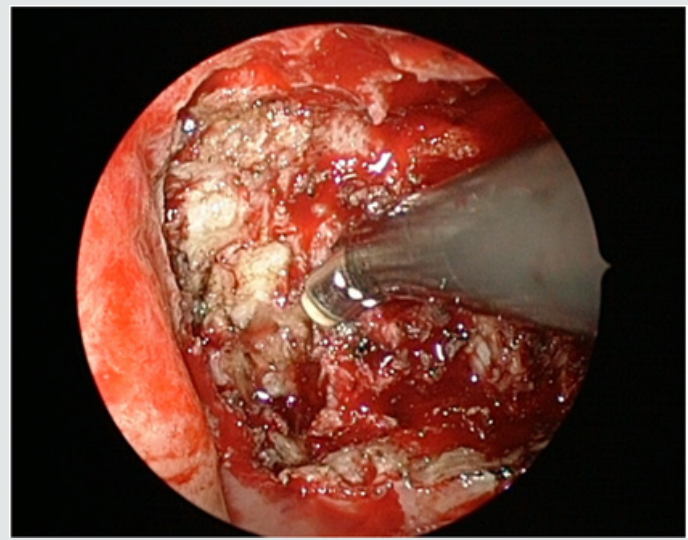

Figure 3: Use of coblator.

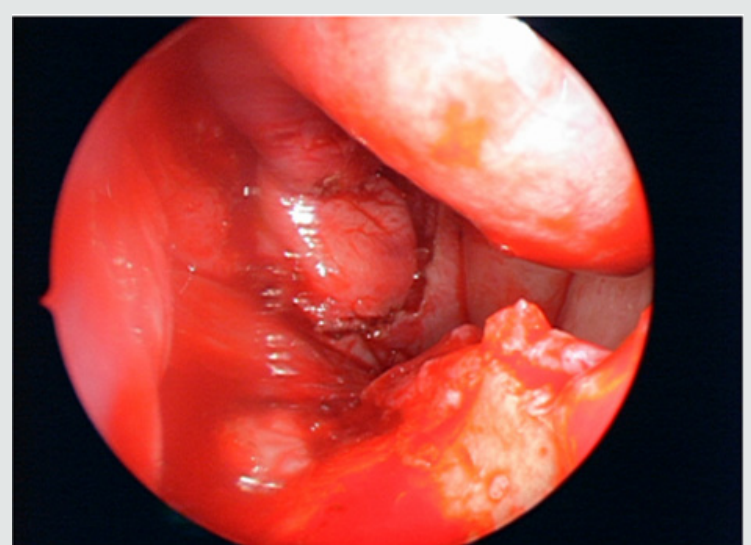

Figure 4: Nasoseptal flap covering nasopharyngectomy wound.

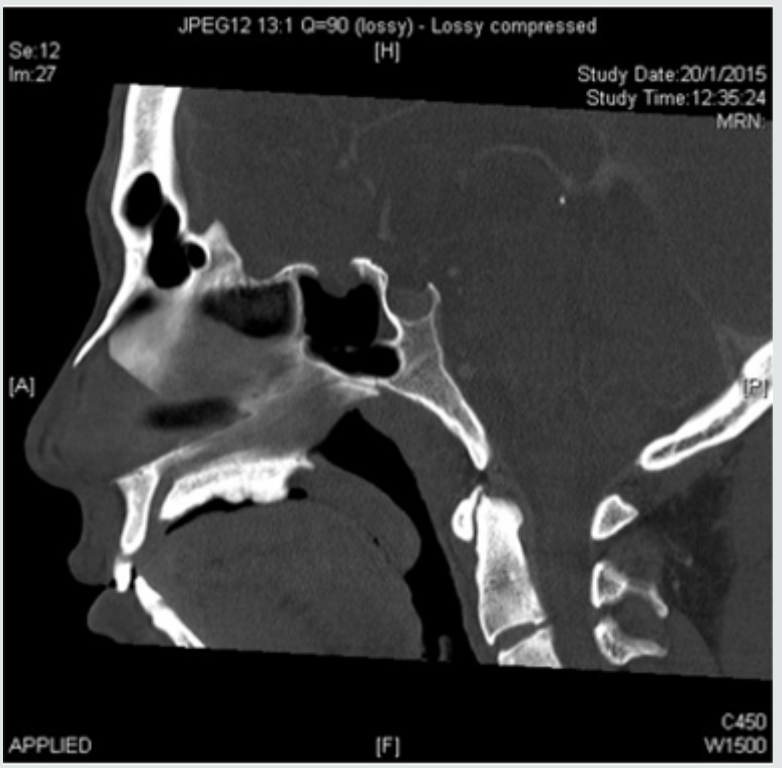

Figure 5: Meningocele.

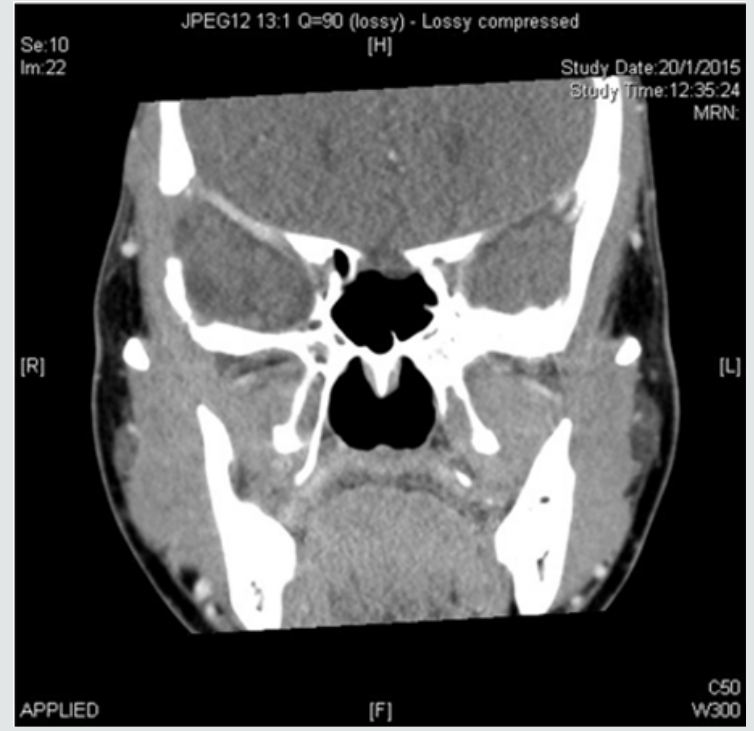

Figure 6. 


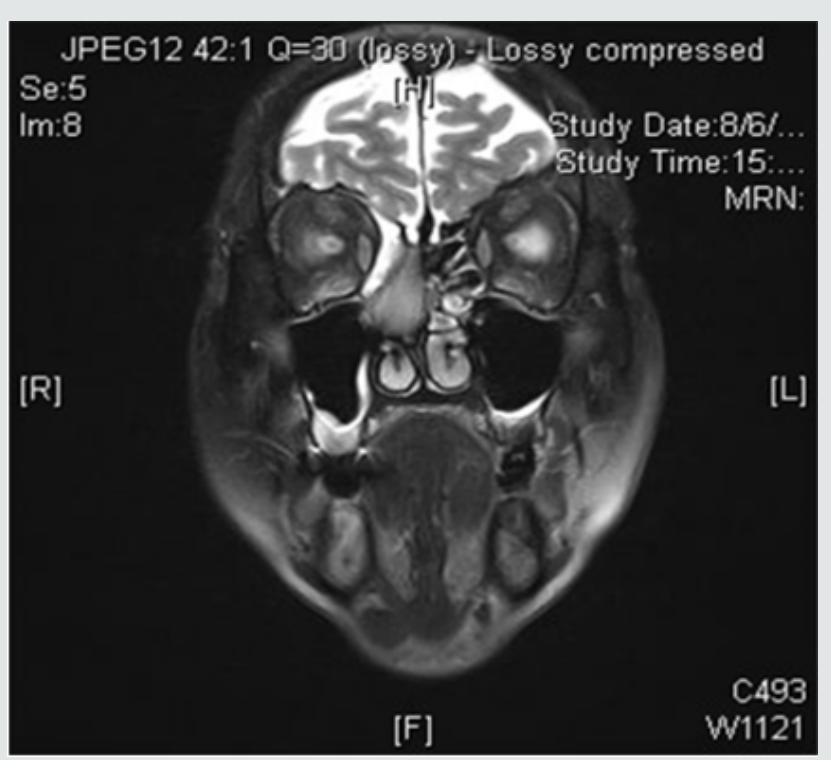

Figure 7: Extracranial neuroblastoma.

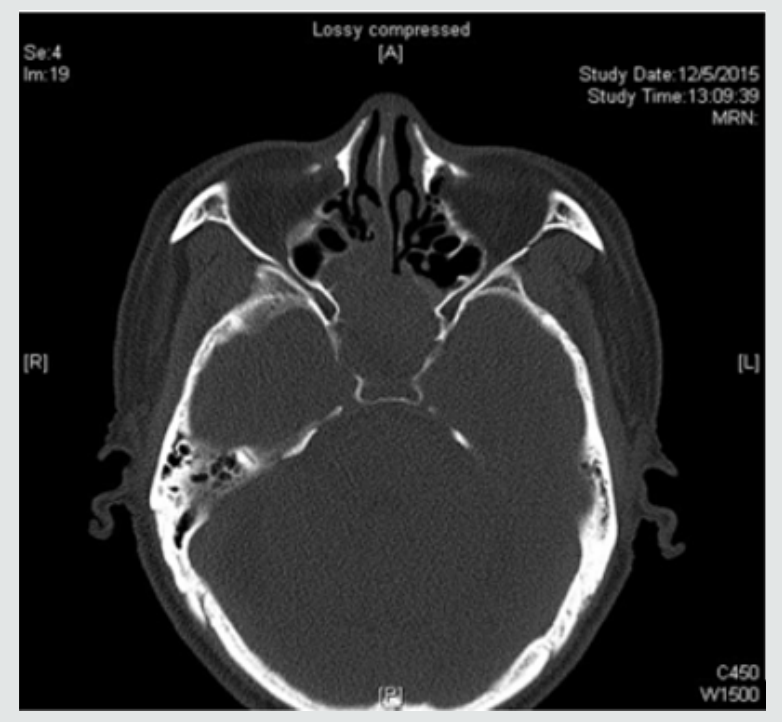

Figure 8: Sphenoid sinus inverted papillom.

The third type of surgery is for tumor of neuroblastoma and inverted papilloma involving skull base. Endoscopic approach with post operation radiotherapy is used for a complete control of two cases of neuroblastoma with no intracranial extension. One inverted papilloma occupied and expands the sphenoid sinus and removed endoscopically (Figures $8 \& 9$ ). The last type of surgery is endoscopic removal of intraconal intraorbital tumor. It was done together with eye doctor to debulk and excise one cavernous haemangioma and one schwannoma (Figure 10). Lastly the degree of difficulty of endoscopic skull base surgery can be classified into different ladder according to some expert (e.g. Prof. Richard Carrau) It starts with level 1 to level 5. Level include sinonasal surgery, level
2 Pituitary gland surgery, CSF leak. Level 3 is extradural surgery of transcribriform to transodontoid etc. Level 4 is intradural and level 6 in cerebrovascular surgery. It takes a great effort to learn the technique in advanced level and a high-risk surgery. A teamwork with neurosurgeon is essential when intradural pathology is managed and therefore not every center even with neurosurgery support can do these surgeries especially with inadequate caseload experience.

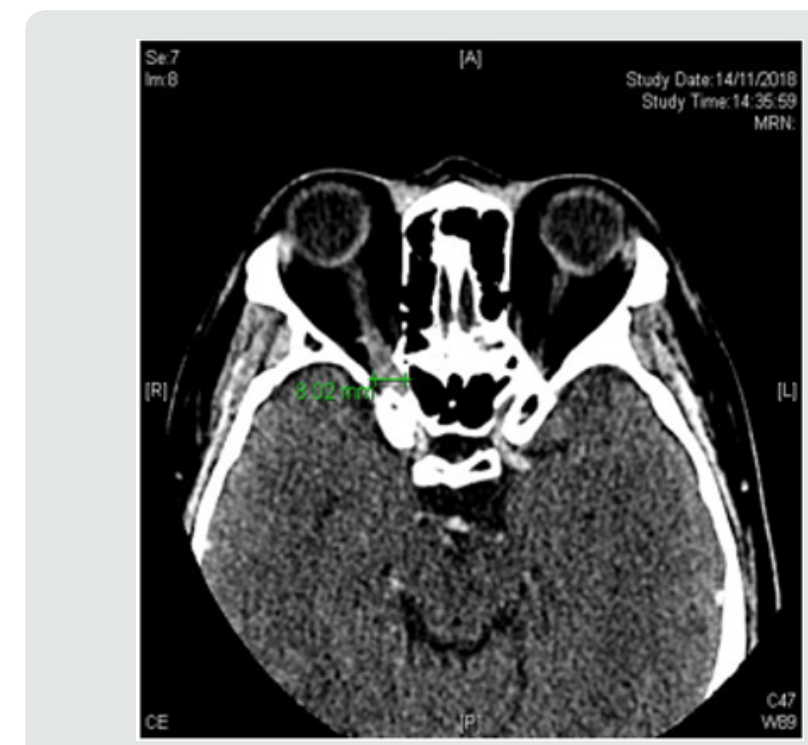

Figure 9.

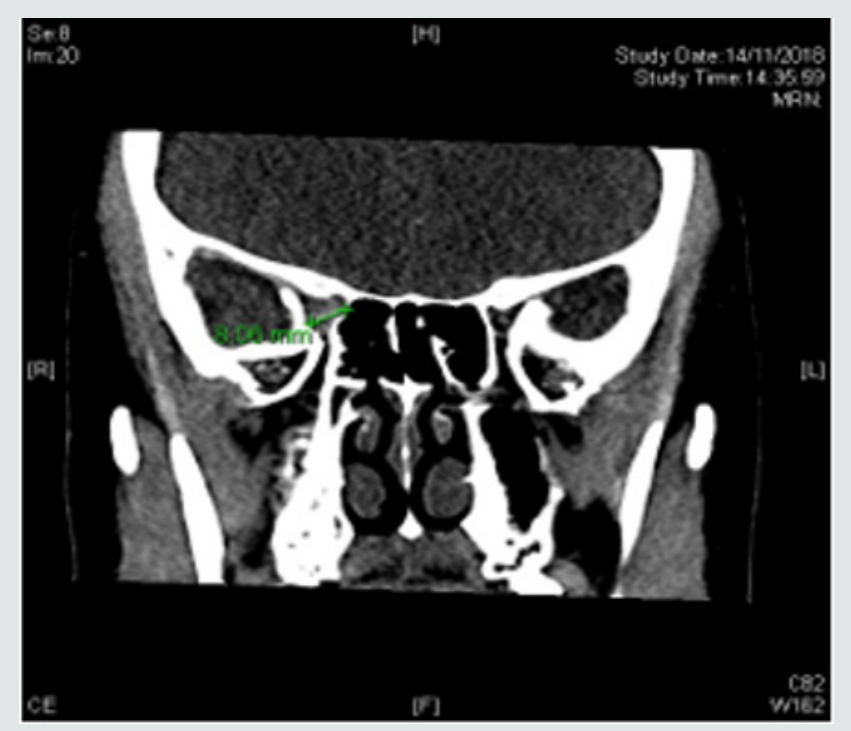

Figure 10.

\section{References}

1. Lau WY, Chong HM, Yu HC, Ngan KC (2012) Endoscopic nasopharyngectomy in salvaging locally recurrent nasopharyngeal carcinoma.

2. HM Chong, PY Lo, RKC Ngan (2015) Endoscopic Nasopharyngectomy for Recurrent Nasopharyngeal Carcinoma. J Radiol 18(1): 37-40. 
This work is licensed under Creative Commons Attribution 4.0 License

\section{To Submit Your Article Click Here: Submit Article}

DOI: $10.32474 /$ SJO.2019.03.000152

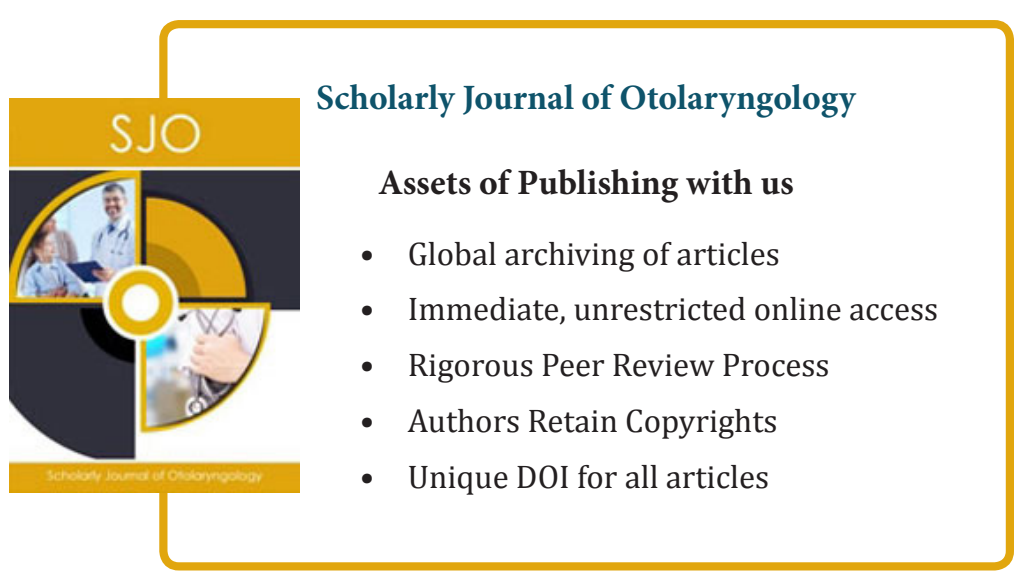

